\title{
Stokes Vector Single-ended Direct Detection Using a 3×3 Coupler Hybrid
}

\author{
Chao Wan ${ }^{(1)}$ \\ (1)State Key Laboratory of Information Photonics and \\ Optical Communications \\ Beijing University of Posts and Telecommunications \\ Beijing, China \\ chao_wan@foxmail.com
}

\author{
Jing $\mathrm{Li}^{(1)}$ \\ (1)State Key Laboratory of Information Photonics and \\ Optical Communications \\ Beijing University of Posts and Telecommunications \\ Beijing, China \\ 729621820@qq.com
}

\begin{abstract}
We propose a scheme to implement the Stokes vector single-ended direct detection (SV-DD) using a $3 \times 3$ coupler hybrid. A 112-Gb/s single-polarization modulated 16-QAM signal is successfully received after $240-\mathrm{km}$ SSMF transmission. SV-DD using $3 \times 3$ couplers may provide a more cost-effective and more power-efficient solution to achieve $100 \mathrm{G}$ short-reach transmission with high spectral efficiency and low system complexity.
\end{abstract}

Keywords-stokes; coupler; polarization; SSMF; OFDM

\section{INTRODUCTION}

The fast growth is driven by the demand for higher data rates for computer connections, data storage, and local communication including linking to internet traffic. Server virtualization, cloud computing, and higher speed ports are now driving networks to $40 / 100-\mathrm{Gb} / \mathrm{s}$ and eventually higher speeds (400G) in data centers.

So short-reach networks within distance of hundreds of kilometers, need to increase their capacity per wavelength beyond 40 or even $100 \mathrm{~Gb} / \mathrm{s}$ [1-5] to meet the everincreasing traffic demand. Different from long-haul communications, these short-reach networks require massive number of transceivers across diverse geographic zones. Therefore, lowering the system cost becomes a priority for short-reach networks. Direct detection (DD), especially a host of self-coherent ( $\mathrm{SCOH}$ ) systems [3-7] is suitable for short-reach applications since they can significantly lower the expense compared with coherent counterpart while achieving both high data rate and moderate reach. However, to remove SSBN, early SCOH systems leave frequency gaps at spectrum to separate signal and the signal-to-signal beat noise (SSBN) [6]. Spectrum efficiency (SE) is sacrificed in this case. Recently, blockwisephase switching (BPS) [4] and signalcarrier interleaved (SCI) [5] DD schemes are proposed to make full use of the frequency band. Some schemes have

\author{
jinnan Zhang ${ }^{(1)} *$ \\ (1)State Key Laboratory of Information Photonics and \\ Optical Communications \\ Beijing University of Posts and Telecommunications \\ Beijing, China \\ zhangjinnan@bupt.edu.cn \\ * Corresponding Author \\ Chenzhao Wei ${ }^{(1)}$ \\ (1)State Key Laboratory of Information Photonics and \\ Optical Communications \\ Beijing University of Posts and Telecommunications \\ Beijing, China \\ 1121170333@qq.com
}

demonstrated dual-polarization modulation and reception $[3,8]$, but with the transmitter complexity approaching POL-MUX coherent systems. Moreover, none of the above-mentioned DD methods achieves $100 \%$ SE, except the approach employing a sophisticated narrow optical filter for optical carrier extraction [9], which can be potentially expensive and difficult to operate. Stokes vector DD is proposed to achieve high SE compared with coherent detection of single-polarization modulation [10]. It only requires one polarization modulation leading to a simpler transmitter structure. Additionally the receiver of SV-DD does not need a local oscillator and $90^{\circ}$ hybrid; the DSP is much simpler due to using less complex algorithm operations, and no need to track laser frequency offset and phase noise.

In this paper, which extends the results of [10], we explore ways to simplify the optical front-end We demonstrate the use of a simple symmetric $3 \times 3$ coupler in conjunction with single-ended photo-detectors (instead of an optical hybrid with two pairs of balanced detectors) and implement the successful reception of 112-Gb/s 16QAM direct-detected signal after transmission over 240-km standard single mode fiber (SSMF) experimentally without electrical pre-compensation or optical compensation.

\section{PRINCIPLE OF StOKes Vector DiRect Detection}

The proposed SV-DD scheme can gracefully resolve two fundamental limitations for direct-detections: (1) chromatic dispersion (CD) induced signal fading due to lack of phase diversity, and (2) 2nd order nonlinearity due to photo-detection. The signal (S) and carrier (C) are respectively placed onto two orthogonal polarizations at the transmitter with a polarization beam combiner (PBC) in the SV-DD scheme [10].

The signal can be expressed by a Jones vector $\mathrm{J}=[\mathrm{S}, \mathrm{C}]^{T}$, where a vector or matrix is represented in bond 
font and superscript ' $T$ ' stands for transpose. Converting the Jones vector to the Stokes space, we arrive at the Stokes vector

$$
S=[S 1, S 2, S 3]^{T}=\left[|S|^{2}-|C|^{2}, \operatorname{Re}\left(S \cdot C^{*}\right), \operatorname{Im}\left(S \cdot C^{*}\right)\right]^{T}
$$

, where $\operatorname{Re}()$ and $\operatorname{Im}()$ represent for real part and imaginary part of a complex number. This Stokes vector can be detected as follows: the signal is split with a polarization beam splitter (PBS) into two outputs of $X$ and $\mathrm{Y}$ respectively. Since the signal polarization has been randomly rotated in the fiber, the received signals $\mathrm{X}$ and $\mathrm{Y}$ are the mixture of the transmitted signals of $\mathrm{S}$ and $\mathrm{C}$.

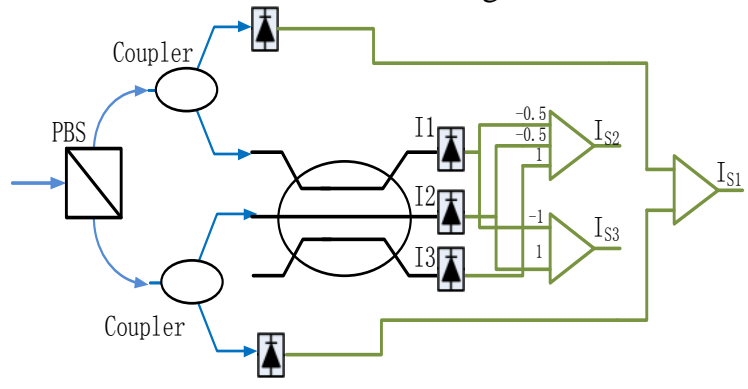

Figure 1. Receiver structure for measuring Stokes vector

It plays key role to acquire this polarization rotation (PR) between the input and out signals in SV-DD scheme. In the coherent detection the PR is accomplished in the Jones space, while in SV-DD it is done in the Stokes space. In particular, we measure the signal Stokes vector (SV) using a DD subsystem shown in Fig. 1.

Fig. 1 shows the architectures of a SV-DD receiver using single-ended photo-detectors (PD) based on $3 \times 3$ couplers. In the architecture shown in Fig. 1, the operations to obtain the $\mathrm{S} 1, \mathrm{~S} 2, \mathrm{~S} 3$ components from the photocurrents are performed with simple analog scaling and subtraction circuits followed by two analog-to-digital converters (ADCs), in analogy to a single-ended. Both $\mathrm{X}$ and $\mathrm{Y}$ are split with $3 \mathrm{~dB}$ couplers, which are identified as ports 1 and 4 respectively. Ports 1 and 4 are fed into single-end PD directly, resulting in the output of $|X|^{2}$ and $|Y|^{2}$ respectively, which can acquire the 1st component of $\mathrm{SV},|X|^{2}-|Y|^{2}$, by simple subtraction circuits or calculation in DSP. Ports 2 and 3 are fed into a symmetric $3 \times 3$ coupler followed by three single PD.

In the receiver proposed here, $S 2=\operatorname{Re}\left(S \cdot C^{*}\right)$ and $S 3=\operatorname{Im}\left(S \cdot C^{*}\right)$ components are obtained from the three detected currents with the following simple operations [11],

$$
\left\{\begin{array}{l}
I_{s 2}=I_{3}-0.5 I_{1}-0.5 I_{2}=\left|S \cdot C^{*}\right| \cos \Delta \theta \\
I_{s 3}=0.5 \sqrt{3}\left(I_{2}-I_{1}\right)=\left|S \cdot C^{*}\right| \sin \Delta \theta
\end{array}\right.
$$

Where $\Delta \theta=\theta_{c}-\theta_{s}$;

Like in a conventional balanced receiver in [11], the direct-detection signal term and the LO term (including the LO relative intensity noise (RIN)) are automatically eliminated by performing the above operations. We obtain the SV described as

$$
S=[S 1, S 2, S 3]^{T}=\left[|S|^{2}-|C|^{2}, \operatorname{Re}\left(S \cdot C^{*}\right), \operatorname{Im}\left(S \cdot C^{*}\right)\right]^{T}
$$

To recover the signal, the remaining task is to acquire the SV rotation matrix (RM) of the channel and rotate the Stokes vectors at receiver back to those at transmitter. This can be done by adding a special training pattern before each signal frame in time domain. We keep the carrier power constant. During the 1st training symbol, no signals are sent; as a result, the Jones vector is $(0,1)$, which corresponds to the SV of $(-1,0,0)$. As shown in Fig. 1(c), the 1st column of SV rotation matrix can be acquired. Similarly, during the 2nd training symbol, we send real signal with constant power same as that of carrier, resulting in the Jones vector of $(1,1)$ and SV of $(0,1,0)$, which can be used to acquire the 2nd column of the RM. We then send Jones vector $(i, 1)$ or SV of $(0,0,1)$ in the 3rd symbol for the training of 3rd column of RM. Similar to channel equalization in coherent systems, we can multiply the received SV with the inverse of the rotation matrix, and the transmitted SV can be recovered as $\left[|S|^{2}-|C|^{2}, \operatorname{Re}\left(S \cdot C^{*}\right), \operatorname{Im}\left(S \cdot C^{*}\right)\right]^{T}$.The $\mathrm{SV}$ rotation matrix (RM) of the channel by adding a special training pattern before each signal frame in time domain [10].

\section{EXPERIMENTAL SETUP}

The SV-DD scheme can be applied to both OFDM and single-carrier systems. The SV-DD scheme is demonstrated experimentally in single-carrier systems. Fig. 2 illustrates the experimental setup.

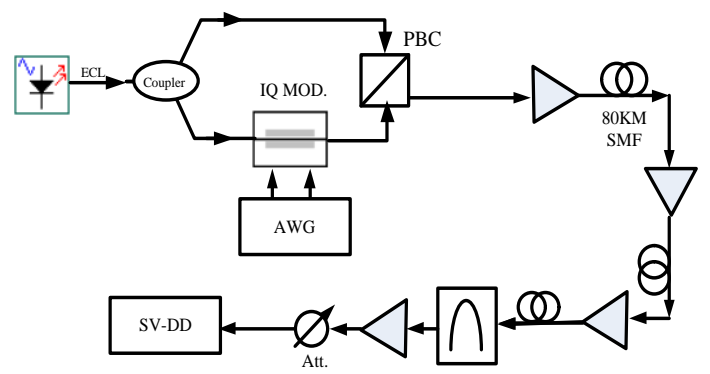

Figure 2. Experimental setup for 112-Gb/s 16-QAM SV-DD

The CW signal of a laser is first split into two branches respectively for the signal and carrier. For the signal branch, the optical signal is into an I/Q modulator driven by an arbitrary waveform generator (AWG). The signal with a 16QAM modulation is loaded into the AWG. SV training symbols are added before signal data with symbol length of 384 points (corresponding to the length of three training symbols mentioned in Part 2). The AWG operates at a sampling rate of $28 \mathrm{GSa} / \mathrm{s}$, resulting in single polarization 16-QAM. The data rate is $112 \mathrm{~Gb} / \mathrm{s}$ [12].

The lower branch is a delay line whose fiber length is matched with the upper line to cancel the phase noise between signal and carrier. Signal carrier power ratio (CSPR) is maintained to be $0 \mathrm{~dB}$. These two branches are combined with a polarization beam combiner (PBC) and then launched into 3-span 80-km SSMF link with an optical amplifier at the end of the each span. After amplified spontaneous emission (ASE) noise loading, the signals are sent into the SV-DD receiver. The electrical 
signal is sampled by a real-time oscilloscope at a sampling rate of $50 \mathrm{GSa} / \mathrm{s}$ with $25-\mathrm{GHz}$ electrical bandwidth. The captured signal was digitally processed offline.

\section{RESULTS AND DISCUSSION}

Polarization modulation is investigated. We measure the bit error rate (BER) as the function of fiber launch power to determine the optimum fiber launch power for 240-km SSMF transmission, as shown in Fig. 3(a).

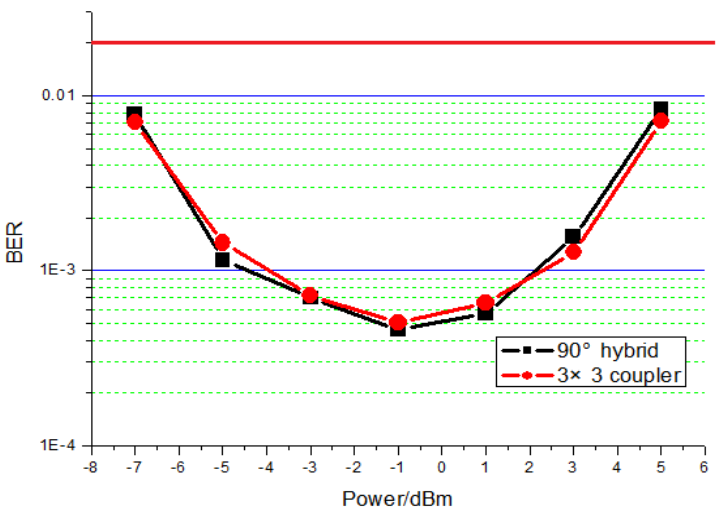

(a)

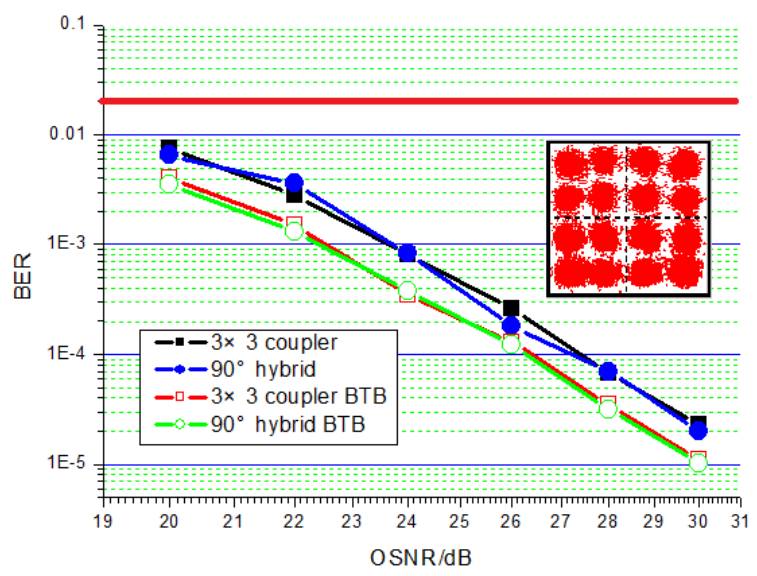

(b)

Figure 3. Bit Error Rate (BER) performance as a function of: (a) fiber launch power; (b) optical signal-to-noise ratio (OSNR).

For 16-QAM single polarization modulation transmission, the optimum launch power is about $-1 \mathrm{dBm}$. Compared with coherent detection, the optimum launch power for both data rate is nearly $3 \mathrm{~dB}$ higher, as mentioned in the literature [10]. This is because half of the launch power is shared by carrier in SV-DD, in other words, the carrier in one polarization just like the LO in coherent detection. Using the optimum launch power, we measure the OSNR sensitivity of the system as shown in Fig. 3(b). To achieve BER below 20\% FEC threshold, SVDD only requires an OSNR of about $20 \mathrm{~dB}$ for $16-$ QAM.After 240-km SSMF transmission, the OSNR is about $0.8 \mathrm{~dB}$ higher than that of back-to-back at BER of 1E-3. The inset is a constellation measured for 16-QAM signal at an OSNR of $26 \mathrm{~dB}$ using $3 \times 3$ coupler. This result indicates the probability for SV-DD with single carrier modulation to achieve higher data rate or longer transmission distance.

We also compared the performance of this receiver with a balanced PD with $90^{\circ}$ hybrids. The results are given in Fig. 3 too. It shows that the single-ended receiver using a $3 \times 3$ coupler as a hybrid can achieve similar performance as the balanced receiver using a full $90^{\circ}$ hybrid.

\section{CONCLUSIONS}

We have demonstrated a SV-DD receiver using simple symmetric $3 \times 3$ couplers for optical hybrids and singleended photo-detectors. 112-Gb/s single polarization 16QAM reception over $240-\mathrm{km}$ SSMF using the SV-DD algorithm is implemented. SV-DD using $3 \times 3$ couplers may provide a more cost-effective and more power-efficient solution to achieve 100G short-reach transmission with high spectral efficiency and low system complexity.

\section{ACKNOWLEDGMENT}

This work is supported by National Key Basic Research Program 973 Project (No. 2013CB329202), 863 Project (No. 2013AA014202), National Natural Science Foundation of China (No. 61571067) and Fund of State Key Laboratory of Information Photonics and Optical Communications (Beijing University of Posts and Telecommunications).

\section{REFERENCES}

[1] N. Cvijetic, et al., "Terabit Optical Access Networks Based on WDM-OFDMA-PON,”JLT. 30, 493-503(2012)

[2] J. L. Wei, et al., "3- $\lambda$ characterization of phase response for optical receivers"ECOC'2012, paper P6.05(2012)

[3] B. Schmidt, et al., "120 Gbit/s Over 500-km Using Single-Band Polarization-Multiplexed Self-Coherent Optical OFDM,'JLT. 28, 328-335, 2010

[4] X. Chen, et al., "Flexible All-Optical OFDM using WSSs,'OFC'2013, paper PDP5B.7,(2013)

[5] X. Chen, et al., "Signal-carrier interleaved optical OFDM for direct detection optical communication,"Opt. Express 21, 32501-32507, (2013)

[6] A. J. Lowery, et al., "Orthogonal-frequency-division multiplexing for dispersion compensation of long-haul optical systems,"Opt. Express 14, 2079-2084, 2006

[7] W. R. Peng, et al., "Spectrally efficient direct-detected OFDM transmission employing an iterative estimation and cancellation technique, "Opt. Express 17, 90[99-9111, 2009

[8] M. Nazarathy, et al., "Doubling Direct-detection Data Rate by Polarization Multiplexing of 16-QAM without a Polarization Controller" ECOC'2013, paper Mo.4.C.4

[9] L. Xu, et al., "Coherent Optical OFDM Systems Using Self Optical Carrier Extraction"OFC'2008, paper OMU4

[10] Di Che et al., "160-Gb/s Stokes Vector Direct Detection for Short Reach Optical Communication, "OFC2014, Th5C.7.(2014)

[11] Chongjin Xie et al., "Colorless coherent receiver using $3 \times 3$ coupler hybrids and single-ended detection, ," Vol. 20, No. 2a, p1164,OPTICSEXPRESS(2012)

[12] Zhang, JN et al., "New control algorithm for automatic PMD compensation system,"JLT.10,030607(2012) 